\title{
Velocity-Dependent Forces in Atomic Force Microscopy Imaging of Lipid Films
}

\author{
J. Rädler, ${ }^{\dagger}$ M. Radmacher, ${ }^{\ddagger}$ and H. E. Gaub* \\ Biophysics E22, Physikdepartment, Technische Universtät München, Munich, Germany \\ Received February 11, 1994. In Final Form: May 17, $1994^{\otimes}$
}

\begin{abstract}
We have imaged adsorbed fluid lipid bilayers by atomic force microscopy. The patches were formed by rupture of phospholipid vesicles onto magnesium fluoride. We show that the membrane patches are fluid but can be stably imaged at scan rates higher than $6 \mu \mathrm{m} / \mathrm{s}$. At lower scan rates the tip penetrates through the layer. The penetrating tip does not destroy the fluid patches, and the previous image can be restored after increasing the scanning velocity. The dynamic forces that possibly explain the effect are discussed.
\end{abstract}

\section{Introduction}

In recent years the atomic force microscope $e^{1,2}$ has evolved into a standard instrument for the investigation of Langmuir-Blodgett (LB) films. ${ }^{3-10}$ Molecular resolution has been obtained on a broad variety of crystalline films; even grain boundaries and point defects were resolved. ${ }^{7}$ LB films have in turn evolved into standard model systems for the improvement of the understanding of fundamental imaging mechanisms, e.g., measuring the elastic properties by atomic force microscopy (AFM). ${ }^{11}$ There are several reasons for this: LB films can be designed such that they are molecularly flat. They can be made from a broad variety of substances, and their molecular packing can be varied and controlled to a large degree at the air-water interface prior to transfer.

By the very nature of the imaging mechanism, considerable forces between the tip and sample are a necessity in AFM imaging. In the case of hard samples like mineral surfaces these forces generally do not lead to any damage. However, in the case of thin organic films like crystalline LB films the minimum forces are just barely small enough to allow stable imaging without significant wear. Special precautions to reduce the imaging forces and to stabilize the films (e.g., with in-plane cross-linking of the molecules) have recently made molecular resolution images of such films possible.

It is also known that a prerequisite for imaging LB films is a strong coupling between the film and the underlying substrate. This problem can be solved either by chemical fixation, e.g., silane on glass, or by taking advantage of a strong interaction between the headgroup of the

* To whom correspondence should be addressed.

${ }^{\dagger}$ Current address: Materials Department, University of California, Santa Barbara, CA 93106.

$\ddagger$ Current address: Department of Physics, University of California, Santa Barbara, CA 93106.

$\otimes$ Abstract published in Advance ACS Abstracts, August 1, 1994.

(1) Binnig, G.; Quate, C. F.; Gerber, C. Phys. Rev. Lett. 1986, 56, 930-933.

(2) Rugar, D.; Hansma, P. K. Phys. Today 1990, 43, 23-30.

(3) Egger, M.; Ohnesorge, F.; Weisenhorn, A.; Heyn, S. P.; Drake, B. Prater, C. B.; Gould, S. A. C.; Hansma, P. K.; Gaub, H. E. J. Struct. Biol. 1990, 103, 89-94.

(4) Hansma, H. G.; Gould, S. A. C.; Hansma, P. K.; Gaub, H. E.; Longo, M.; Zasadzinski, J. A. N. Langmuir 1991, 7, 1051-1054.

(5) Goettgens, B. M.; Tillmann, R. W.; Radmacher, M.; Gaub, H. E. Langmuir 1992, 8, 1768-1774.

(6) Tillmann, R. W.; Radmacher, M.; Gaub, H. E.; Kenny, P.; Ribi, H. O. J. Phys. Chem., in press.

(7) Garnaes, G.; Schwartz, D. K.; Viswanathan, R.; Zasadzinski, J. A. N. Nature $1992,257,508-511$.

(8) Heckl, W. Thin Solid Films 1992, 210/211, 640-647.

(9) Bordieu, L.; Silberzan, P.; Chatenay, D. Phys. Rev. Lett. 1991,67, 2029-2032.

(10) Singh, S.; Keller, D. J. Biophys. J. 1991, 60, 1401-1410.

(11) Radmacher, M.; Tillman, R. W.; Gaub, H. E. Biophys. J. 1993, $64,735-742$. amphiphiles and the substrate (e.g., cadmium arachidate on glass). These considerations led to the assumption that fluid and weakly fixed films cannot be imaged by AFM. In fact it was shown that in the case of fluid films the tip penetrated in a static approach. ${ }^{8,12,13}$ These films show also the amazing property of self-healing after disrupture, which has been demonstrated by cooling the films below the transition temperature and heating them above it again. ${ }^{14}$

On the other hand, AFM gained an enormous importance in biology, when it became clear that cell surfaces can be imaged..$^{15-20}$ The resolution of about $50 \mathrm{~nm}$ that is achieved on living cells is remarkable because biological membranes are at least partly fluid and allow rapid lateral movement of lipids and membrane-associated proteins. Even with the extracellular matrix and parts of the cytoskeleton supporting the membrane, ${ }^{16}$ the actual scanning mechanism is not obvious and a detailed picture of the impact of the atomic force microscope tip on the cell surface can not be given yet.

In this study patches of synthetic lecithin bilayers were chosen as a test sample. Fluid patches of lipid bilayers can be produced by surface-induced vesicle fusion. In this technique vesicles are added in aqueous suspension onto a suitable surface. The vesicles adhere and flatten out at the surface. This flattening is driven by the attractive interaction between the lipid membrane and the solid support. Eventually the vesicles rupture and spread out to a membrane patch in a wetting-like process. On hydrophilic surfaces a water film due to strong hydration forces generally resides between the film and substrate, resulting in a mechanical decoupling of the lipid bilayer and the solid support. This decoupling allows the lipid film to stay in the fluid phase with lipid diffusion constants slightly smaller than for free membranes. ${ }^{14,21}$

In this paper we present evidence that patches of lecithin bilayers adsorbed onto a magnesium fluoride surface can

(12) Helm, C. A.; Israelachvili, J. N.; McGuiggan, P. M. Science 1989 $246,919-922$.

(13) Ducker, W., submitted for publication

(14) Tamm, L. K.; McConnell, H. M. Biophys. J. 1985, 47, 105-113.

(15) Häberle, W.; Hörber, J. K. H.; Ohnesorge, F.; Smith, D. P. E.; Binnig, G. Ultramicroscopy 1992, 42-44, 1161-1167.

(16) Fritz, M.; Radmacher, M.; Petersen, N.; Gaub, H. E. J. Vac. Sci. Technol., in press.

(17) Keller, D.; Chang, L.; Luo, K.; Singh, S.; Yorganciogla, M. Proc. SPIE 1992, 1639, 91-101.

(18) Radmacher, M.; Tillmann, R. W.; Fritz, M.; Gaub, H. E. Science 1992, 257, 1900-1905.

(19) Henderson, E.; Haydon, P. G.; Sakaguchi, D. S. Science 1992, $257,1944-1946$.

(20) Fritz, M.; Radmacher, M.; Gaub, H. E. Biophys. J. 1994, 66, $1328-1334$.

(21) Merkel, R.; Sackmann, E.; Evans, E. J. Phys. (Paris) 1989, 50, 1535-1555. 

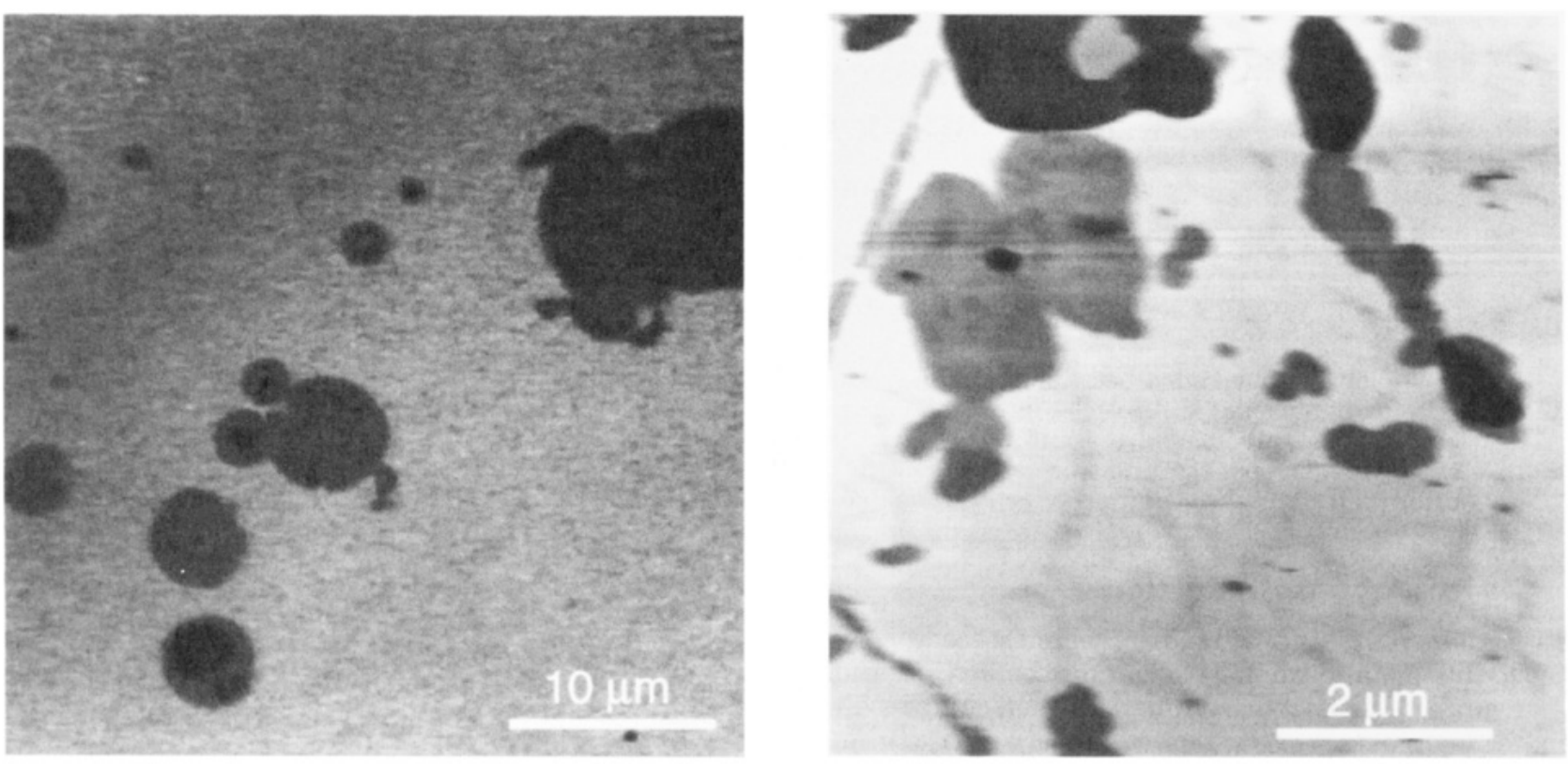

A

Figure 1. (A) DMPC vesicles ruptured and spread out to patches on a magnesium fluoride covered glass surface observed with optical reflection interference microscopy. (B) A sample prepared in the same way as that of (A) scanned with the atomic force microscope. In this image the contrast is inverted compared to the usual convention for AFM images, so dark shades of gray correspond to higher areas of the sample. This was done to show the similar appearance of the AFM image and the above optical image. Height steps correspond to one, sometimes two, lipid bilayer.

be imaged by AFM even in their fluid state. The contrast of the image, however, appears to be scan rate dependent. We can distinguish two completely different imaging regimes: at high scan rates the bilayer can be scanned for hours and appears with the right height in the topography image. Below a critical scan rate the image is abruptly lost and the apparent contrast is mostly determined by lateral forces. Since we are operating safely below the scan rate limit of the cantilevers in use, the observed effect is due to the sample. ${ }^{22}$ Scan rate dependent lateral forces have been reported recently. ${ }^{23}$ We did not check whether in our case the lateral forces are also dependent on the scan rate, but they might be. We will show that this behavior cannot be explained by modeling the membrane as an isotropic fluid but it is rather a dynamic effect due to the fluid crystalline nature of the lipid bilayer. The appearance of normal stress components (perpendicular to the shearing flow field) are well known for polymeric solutions, melts, ${ }^{24}$ and liquid crystalline phases. ${ }^{25}$

\section{Materials and Methods}

Synthetic lecithin was purchased from Avanti Polar Lipids (Birmingham, AL). Giant vesicles made of DMPC (L- $\alpha$-dimyristoyl-1,2-diacyl-sn-glycero-3-phosphocholine) were prepared as follows. Lipid was dissolved in a stock solution of $10 \mathrm{mg} / \mathrm{mL}$ chloroform/methanol (2:1). A 40-mL sample of the solution was dried on a Teflon disk and desiccated under vacuum for $2 \mathrm{~h}$. Subsequently, bidistilled water was added into a glass beaker with the Teflon disk and left for $24 \mathrm{~h}$ at $40{ }^{\circ} \mathrm{C}$. The stack of membranes swells, and closed vesicles detach from the Teflon surface.

Thin films of magnesium fluoride on glass coverslips were prepared by vapor deposition. The glass was previously cleaned in $2 \%$ Hellmanex solution (Hellma, Germany). The deposition was carried out at a pressure of $10^{-6} \mathrm{~atm}$ with a deposition rate of about $1 \mathrm{~nm} / \mathrm{s}$. The substrate stage with the glass coverslip was temperature controlled and heated to a temperature of 380 ${ }^{\circ} \mathrm{C}$. Typically $200 \AA$ of $\mathrm{MgF}_{2}$ was deposited.

Several milliliters of the vesicle suspension was spread onto the $\mathrm{MgF}_{2}$-coated coverslip. After 2 min the suspension was exchanged with bidistilled water and the coverslip mounted into the atomic force microscope. The AFM experiments were conducted at room temperature $\left(27^{\circ} \mathrm{C}\right)$. For the AFM experiments a homebuilt atomic force microscope $\mathrm{e}^{26}$ as well as a commercial atomic force microscope (Nanoscope III, Digital Instruments, Santa Barbara, $\mathrm{CA}$ ) was used. Independently, the adsorbed vesicles were studied with reflection interference microscopy. The details of this optical investigation that allows the optical thickness of the patches to be measured are described in ref 27.

Fluorescence recovery after photobleaching experiments (FRAP) were performed with a specially configured fluorescence microscope. The experimental setup was described earlier. ${ }^{21}$ Vesicles were prepared as above but with addition of $1 \%$ (7nitrobenz-2-oxa-1,3-diazol-4-yl)dimiristoylphosphatidylcholine (NBD-DMPE) (Avanti Polar Lipids, Birmingham, AL) as a fluorescent probe. $\mathrm{AMgF}_{2}$-covered glass coverslip was incubated with a vesicle suspension and washed with bidistilled water. The patches of ruptured vesicles spread on the $\mathrm{MgF}_{2}$ surface were observed under the fluorescence microscope. To reduce the effects of finite diffusion boundaries, the FRAP experiments were focused on large patches of about 20- $\mu \mathrm{m}$ diameter and a bleaching spot of $2-\mu \mathrm{m}$ diameter was used.

\section{Results}

DMPC vesicles that contact a $\mathrm{MgF}_{2}$ surface rupture and spread out to a single bilayer patch. It was reported earlier that the lipid bilayer can be conventionally visualized with reflection interference microscopy as shown in Figure 1A. ${ }^{28}$ We observed several ruptured or adhered vesicles with the contour of the patches remaining

(22) Butt, H.-J.; Siedle, P.; Seifert, K.; Fendler, K.; Seeger, T.; Bamberg, E.; Weisenhorn, A. L.; Goldie, K.; Engel, A. J. Microsc. 1993, $169,75-84$.

(23) Thundat, T.; Zheng, X.-Y.; Chen, G. Y.; Warmack, R. J. Surf. Sci. Lett. 1993, 294, L939-L943.

(24) Byron, R. B.; Armstrong, R. C.; Hassager, O. Dynamics of Polymer Liquids, 2nd ed.; New York, 1987; Vol. 1.

(25) Bruinsma, R. F.; Safinya, C. R. Phys. Rev. A 1991, 43, 5377.

(26) Radmacher, M.; Eberle, K.; Gaub, H. E. Ultramicroscopy 1992, $42-44,968-972$.

(27) Rädler, J.; Sackmann, E. J. Phys. II 1993, 3, 727-748.

(28) Rädler, J.; Sackmann, E. Langmuir 1992, 8, 848-853. 


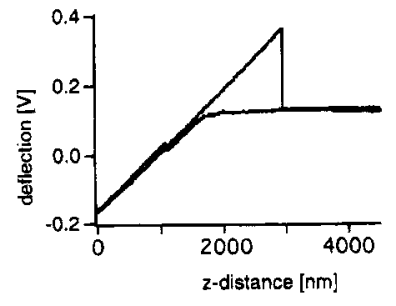

A

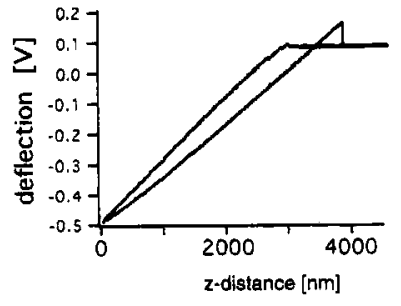

Figure 2. Force scans on the substrate magnesium fluoride (A) and the lipid bilayers (B). The large hysteresis during retract on the magnesium fluoride shows that a strong adhesion is acting between the tip and the substrate. On the contrary the adhesion on the lipid (B) is lower. The force scan on the lipid shows the effects of lateral forces and elasticity of the sample (see text for details).

stable. An evaluation of the optical thickness of the adsorbed membranes resulted in a bilayer thickness of $3.8 \pm 0.2 \mathrm{~nm}$.

An equally prepared sample of adsorbed DMPC vesicles was investigated by AFM. A similar pattern was found as depicted in Figure 1B (note the different scales and the inverted gray scale for the AFM picture). The height profiles yielded steps of typically $5 \mathrm{~nm}$. Usually some structures remained that appeared to be twice as thick as one bilayer patch or more. These structures, however, were less stable and tended to rupture during scanning. The measured step height was somewhat larger than the optically measured membrane thickness.

Force curves show a huge difference in the interaction between the tip and substrate compared to the interaction between the tip and lipid membrane (Figure 2). Adhesion appears in the force curves as a hysteresis during retract which shows the amount of force needed to lift the tip off the surface. The difference in slopes of the contact lines upon approach and retract might be due to lateral forces..$^{29}$ Because of technical reasons, the cantilever is mounted at a small angle, typically $15-20^{\circ}$. Therefore, the cantilever is mainly pushed vertically during the force curve, but the movement also has a lateral component. The other effect, visible in these curves, is a slight deviation from a linear relationship between deflection and sample height in the contact region. This might be explained by an elastic response of the sample. Because both effects are possible with our substrate, we could not separate or quantify them.

We then performed a sequence of experiments where we scanned the same membrane patch with the same magnification but at different scan speeds. The result is given in Figure 3. The sequence clearly shows that at scan speeds above $6 \mu \mathrm{m} / \mathrm{s}$ the contrast between the fluid membrane patches and the substrate is independent of the scan speed and that subsequent images of the same area show no significant differences, which means that no significant wear occurs under these conditions. Below a scan speed of $6 \mu \mathrm{m} / \mathrm{s}$, however, the contrast between the membrane patches and the substrate decreases drastically. In this case a weak image of the patch appeared that was inverted for forward and backward scans, indicating a significant contribution of lateral forces to the apparent contrast. ${ }^{18,30}$ An increase of the scan speed, however, restored the original contrast, and no significant change in the image occurred compared to the preceding fast scans. This switching between fast and slow scanning could be repeated and resulted in qualitatively equivalent images. This means that the loss in contrast was not

(29) Hoh, J. H.; Engel, A. Langmuir 1993, 9, 3310-3312.

(30) Thundat, T.; Warmack, R. J.; Allison, D. P.; Bottomley, L. A. Lourenco, A. J.; Ferrell, T. L. J. Vac. Sci. Technol. 1992, A10, 630-635. caused by a change in the surface topology (like a loss of the membrane patch) but that the imaging mechanism is different at low and at high scan speeds. It should be noted here that this transition as a function of the scan rate between high and low contrast is rather sharp and had, in our measurements, a width of less than $1 \mu \mathrm{m} / \mathrm{s}$.

To examine the fluidity of the bilayer, we performed FRAP experiments on large patches. Figure 4 shows a typical recovery of the fluorescence intensity after photobleaching. The recovery shows that the lipid molecules are in a fluid state and laterally mobile. The data evaluation yields a diffusion constant of $1.3 \pm 0.7 \mu \mathrm{m}^{2} / \mathrm{s}$ with a relative recovery of $85 \%$. The large scatter in the measured mobilities might be due to the different sizes of the patches. The mobility is smaller by a factor of 3 than the known value for a fluid-free DMPC membrane. ${ }^{21}$ These data are consistent with the assumption that the lower monolayer is strongly coupled to the $\mathrm{MgF}_{2}$ surface. The low recovery indicates that about $30 \%$ of the substrateadjacent headgroups are immobile, possibly due to binding of the phosphate group with the divalent $\mathrm{Mg}^{2+}$ ions.

\section{Discussion}

Our experiments showed that fluid membranes can be imaged stably at high scan rates with an apparent height of $5 \mathrm{~nm}$. At lower scan rates the images recorded during trace and retrace were inverted in contrast. The effect of contrast inversion in AFM, depending on scan direction, is reported in the literature and explained to be due to lateral forces. ${ }^{18,30} \mathrm{~A}$ simple explanation for the velocitydependent friction is a penetration of the tip through the membrane patch at lower scan rates in contrast to a sliding on top of the patch resulting in the images at high scan rates.

Let us picture the scanning process as depicted in Figure 5. The lipid headgroups are depicted as circles, with hydrocarbon chains ordered in the smectic A phase. However, in a static approach of the atomic force microscope cantilever we assume the membrane to respond as an oil-like isotropic liquid. The membrane is deformed through the indentation of the tip that is approximated by a sphere with radius $R_{s}$. Hence, the surface tension $\sigma$ of the bilayer will exert a vertical force on the cantilever, which is given by the change in area, i.e., the difference of the surface of a spherical cap with respect to the flat lipid surface, $A_{\mathrm{c}}=A_{\text {sph }}-A_{\text {flat }}=\pi h^{2} . h$ denotes the depth of indentation. The force is then given by

$$
F_{\mathrm{S}}=\sigma\left(\partial A_{\mathrm{c}} / \partial h\right) \approx \sigma 2 \pi h
$$

Estimating a surface tension of the membrane of $\sigma \approx$ $30 \mathrm{mN} / \mathrm{m}$ and $h \approx 1 \mathrm{~nm}$, this static force turns out to be $F \approx 0.2 \mathrm{nN}$. Since the adhesion forces between the tip and the sample are on the order of several nanonewtons, this static force is not sufficient to support the tip, which has also been reported by other researchers. ${ }^{31}$ This argument raises the question of what other forces can support the cantilever during imaging at high scan rates.

We consider now the dynamic case. A simple analogy for the observed phenomena is water skiing. In water skiing the ski has to move faster than the water surface can rearrange. In our case the tip has to be scanned faster than the molecules can rearrange by thermal diffusion. The typical diffusion time that is needed to close a hole of radius $R_{\mathrm{c}}$ created by the scanning tip can be ap-

(31) Specht, M.; Ohnesorge, F.; Heckl, W. M. Surf. Sci. 1991, 257, L653-L658. 


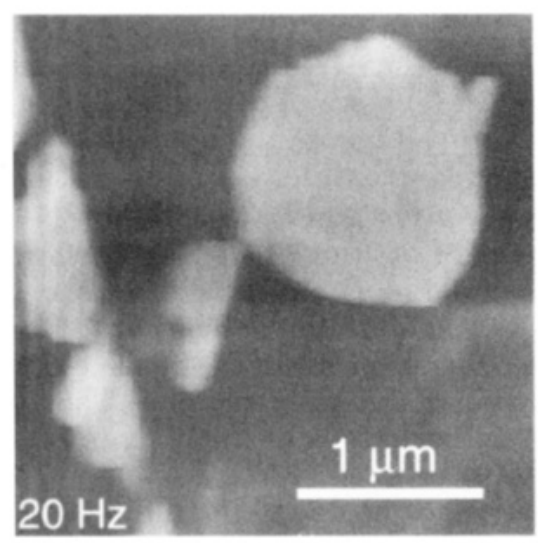

A

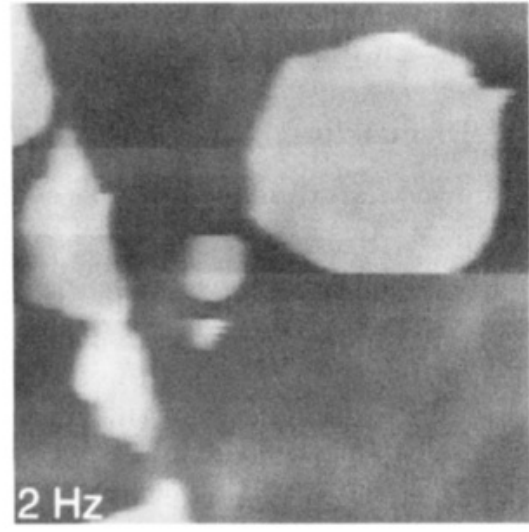

D

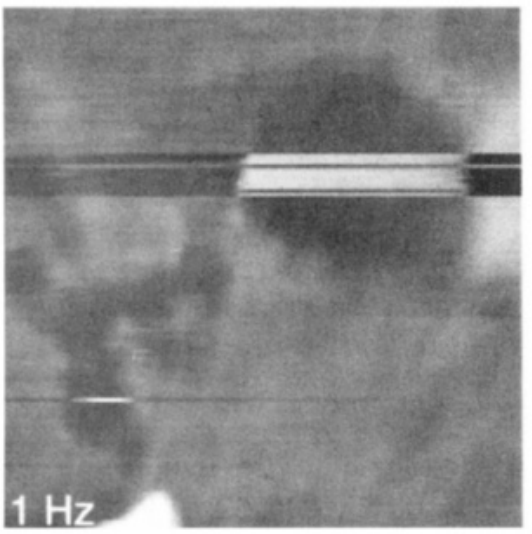

G

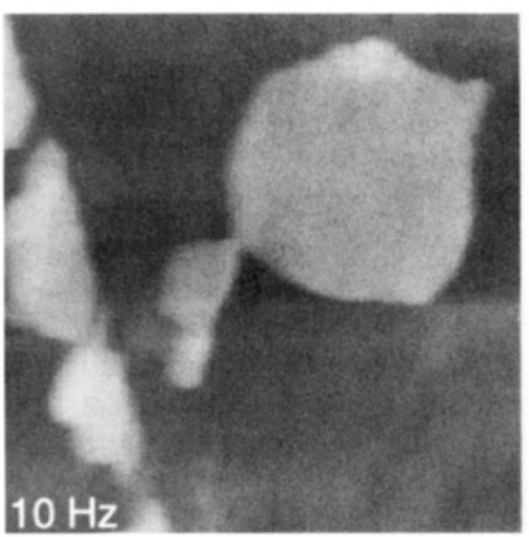

B

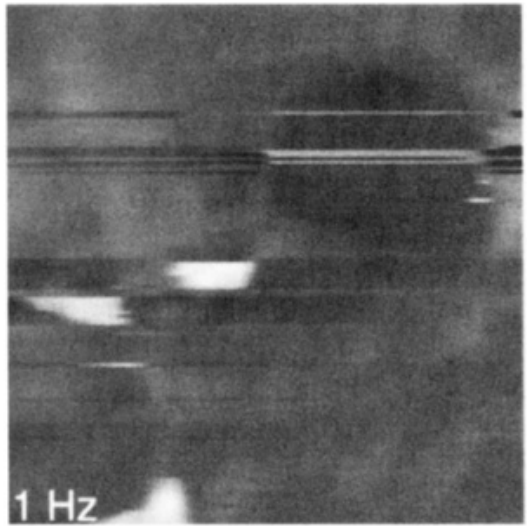

E

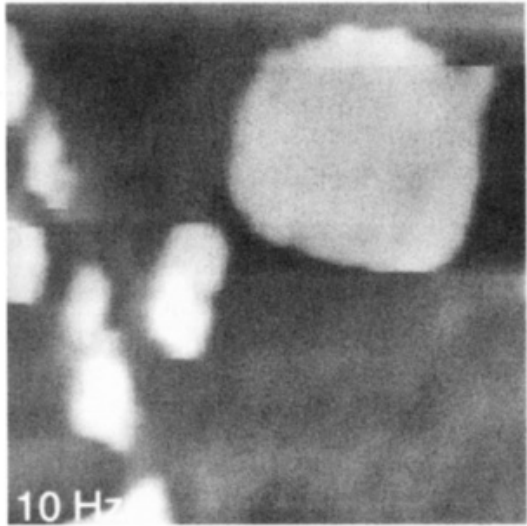

H

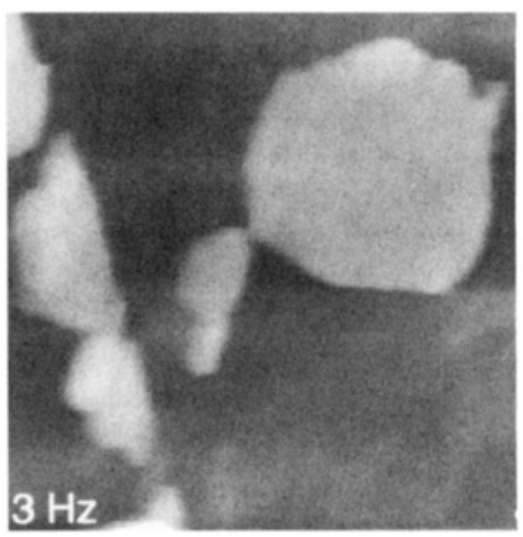

C

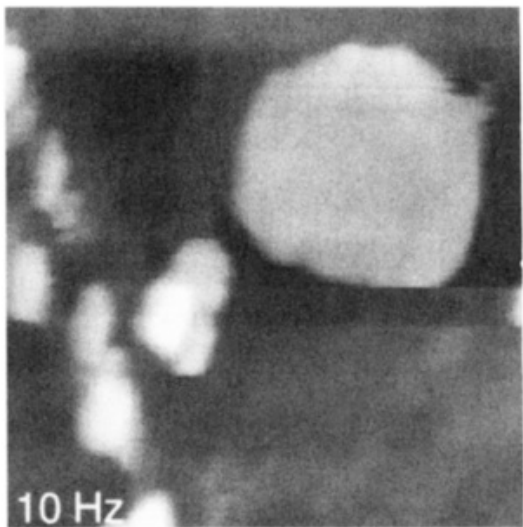

F

Figure 3. Sequence of subsequent AFM images of the same bilayer patch with different scan speeds. The lipid patch could be imaged stably at high scan rates (see (A)-(D)), but suddenly changed its appearance at low scan rates (below $1 \mathrm{~Hz}$, corresponding to a scan speed of $6 \mu \mathrm{m} / \mathrm{s}$; see (E)). In this case the trace and retrace (not shown here) images showed inverted contrast, which indicates high lateral forces. After increasing the scan frequency again, the image of the bilayer patch is regained without significant wear (see $(\mathrm{F})$ and $(\mathrm{H})$ ). (All images show trace scans; from each line the offset and slope have been subtracted to correct for sample tilt.)

proximated by:

$$
\tau \approx\left(\pi R_{\mathrm{c}}\right)^{2} / D_{\mathrm{t}}
$$

A typical contact radius is $R_{\mathrm{c}} \approx 10 \mathrm{~nm}$ for a tip with tip radius $R_{\mathrm{t}} \approx 20 \mathrm{~nm}$ that is penetrating $4 \mathrm{~nm}$ through the membrane. Given $D_{\mathrm{t}} \approx 1 \mu \mathrm{m}^{2} / \mathrm{s}$ we obtain $\tau \approx 10^{-3} \mathrm{~s}$. A critical velocity might be obtained by demanding that the hole created by the tip should heal at the same period of time that it needs to scan through it. Therefore, $v_{\mathrm{c}} \approx R_{\mathrm{d}} d$, resulting in a typical critical velocity of $10 \mu \mathrm{m} / \mathrm{s}$. This number is on the same order as the observed critical scan velocity of $6 \mu \mathrm{m} / \mathrm{s}$ where the sudden change of the imaging process is observed. At scanning velocities below $v_{\mathrm{c}}$, the tip penetrates the membrane and the lipid molecules can rearrange and heal very quickly behind the scanning tip. The estimation also shows that the rearrangement hap- 


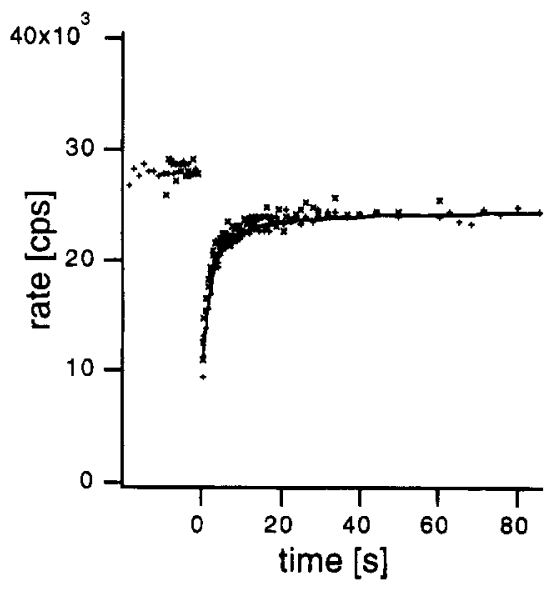

Figure 4. Recovery of the fluorescence of a labeled lipid patch after photobleaching. The experiment was carried out on a large $20-\mu \mathrm{m}$ patch of bilayer. The high mobility indicates that the bilayer is in the fluid state.

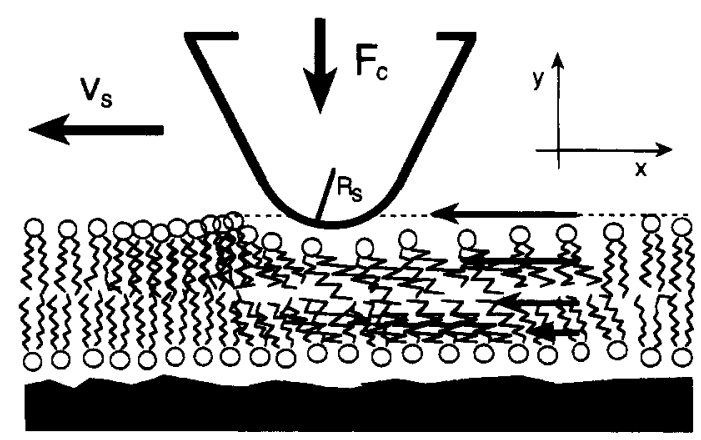

Figure 5. Schematical sketch of the molecular motion during the scan. The tip induces high shear rates that lead to a vertical force component due to shear and condensation of the lipid tails.

pens on a time scale too fast to be directly observed during the scanning process.

On the other hand, if the scanning velocity exceeds $v_{c}$, the molecules cannot be replaced in time. In this case the following picture is more adequate. Figure 5 illustrates how the lipid molecules are tilted and stretched during the scanning process. The local density of molecules in front of the moving tip will be higher than behind the tip. These molecular distortions will lead to elastic forces on small time scales. We may now compare the shear rate $\dot{\gamma}$ to the largest relaxation time $\tau$ of the material under shear. The product $\gamma \tau$ is often called the Deborah number and used as a measure for the onset of the elastic behavior of fluids. ${ }^{24,25}$ The shear rates induced by the scanning tip are extremely large. Since the membrane has a thickness of only $4 \mathrm{~nm}$, we obtain shear rates

$$
\dot{\gamma}=v_{\mathrm{s}} / d_{\mathrm{M}}
$$

on the order of $2 \times 10^{2}-2 \times 10^{4} \mathrm{~s}^{-1}$ for scanning velocities $v_{\mathrm{s}} \approx 1-100 \mu \mathrm{m} / \mathrm{s}$.

As the typical relaxation time we use the above estimated time that a lipid molecule needs to diffuse across the contact area of the scanning tip: $10^{-3} \mathrm{~s}$. The product $\gamma \tau$ turns out to be $0.2-20$. We are therefore crossing over from the fluid-like into the elastic regime. In this high shear rate regime forces normal to the shearing flow field are known to appear in polymeric solutions and melts. ${ }^{24}$ This non-Newtonian flow behavior is also observed in liquid crystalline phases. ${ }^{25}$ Therefore, the liquid crystalline nature of the lipid membrane is crucial to explain its elastic response at higher shear rates. Interestingly the Deborah number for our observed critical velocity is close to unity.

From our experiments the transition appears to be very sharp. A closer look at Figure $3 \mathrm{e}, \mathrm{g}$ reveals that the image is partially striped, exhibiting random changes from imaging the height profile to scanning through the patch. The coexistence of these two different line scans is an indication of an abrupt transition between the two imaging modes. In this case the conditions at the edge of the membrane patch might determine wether a line can be imaged or will be penetrated.

We conclude that fluid membranes can be imaged by AFM due to dynamic normal forces at high scan speeds, while at low scan speeds the tip penetrates through the membrane, giving rise to friction images. Further experiments are needed to clarify the dependencies of the dynamic forces in fluid membranes at very high shear rates. The outstanding properties of surfactant films are of large technological importance for the lubrication of miniaturized mechanical devices. ${ }^{32}$ So far the lateral and vertical force components have not been measured separately. Atomic force microscopy might prove a valuable tool in clarifying the viscoelastic properties of thin films. This will also include films of polymer and proteins for which similar behavior can be expected.

Acknowledgment. We enjoyed helpful discussions with E. Evans and R. Bruinsma. We thank M. Kühner for help with the FRAP measurements and J. Cleveland for carefully revising the paper. We thank one reviewer for drawing our attention to the water skiing analogy.

(32) Yoshizawa, H.; McGuiggan, P. J. I. Science 1993, 259, 1305. 